\title{
MODELING COMPETITION BETWEEN TWO PHARMACEUTICAL DRUGS USING INNOVATION DIFFUSION MODELS -SUPPLEMENTARY MATERIALS-
}

\author{
By Renato Guseo and Cinzia Mortarino* \\ University of Padova, Italy
}

Appendix 1. Proof. Let $z(t)=z_{1}(t)+z_{2}(t)$ denote the sum of the cumulative sales of the two products and $z^{\prime}(t)$ the total instantaneous sales. If we sum the equations of system (3.1), we obtain

$$
\begin{aligned}
z^{\prime}(t)= & z_{1}^{\prime}(t)+z_{2}^{\prime}(t) \\
= & m(t)\left[\left(p_{1}+p_{2}\right)+\left(q_{1}+q_{2}\right) \frac{z_{1}(t)}{m(t)}+\left(q_{1}+q_{2}\right) \frac{z_{2}(t)}{m(t)}\right]\left[1-\frac{z(t)}{m(t)}\right] \\
& \quad+\left[z_{1}(t)+z_{2}(t)\right] \frac{m^{\prime}(t)}{m(t)} \\
(\mathrm{A} 1.1)= & m(t)\left[\left(p_{1}+p_{2}\right)+\left(q_{1}+q_{2}\right) \frac{z(t)}{m(t)}\right]\left[1-\frac{z(t)}{m(t)}\right]+z(t) \frac{m^{\prime}(t)}{m(t)} .
\end{aligned}
$$

Equation (A1.1) defines a coevolutive model (Guseo and Guidolin, 2009) with unspecified market potential $m(t)$ and adoption parameters $p_{s}=p_{1}+p_{2}$ and $q_{s}=q_{1}+q_{2}$. Thus, the solution of the differential equation (A1.1), with initial condition $z(0)=0$, is given below:

$$
\frac{z(t)}{m(t)}=w(t)=\frac{1-e^{-\left(p_{s}+q_{s}\right) t}}{1+\frac{q_{s}}{p_{s}} e^{-\left(p_{s}+q_{s}\right) t}} .
$$

In order to find a solution for $z_{1}(t)$, the first equation in system (3.1)

${ }^{*}$ Corresponding author 
should be rearranged in the following manner:

$$
\begin{aligned}
z_{1}^{\prime}(t)-z_{1}(t) \frac{m^{\prime}(t)}{m(t)} & =m(t)\left[p_{1}+\left(q_{1}+\delta\right) \frac{z_{1}(t)}{m(t)}+q_{1} \frac{z_{2}(t)}{m(t)}\right]\left[1-\frac{z(t)}{m(t)}\right] \\
\frac{z_{1}^{\prime}(t) m(t)-z_{1}(t) m^{\prime}(t)}{m^{2}(t)} & =\left[p_{1}+q_{1} \frac{z(t)}{m(t)}+\delta \frac{z_{1}(t)}{m(t)}\right]\left[1-\frac{z(t)}{m(t)}\right] \\
{\left[\frac{z_{1}(t)}{m(t)}\right]^{\prime} } & =\left[p_{1}+q_{1} \frac{z(t)}{m(t)}+\delta \frac{z_{1}(t)}{m(t)}\right]\left[1-\frac{z(t)}{m(t)}\right] \\
(\mathrm{A} 1.3) \quad\left[\frac{z_{1}(t)}{m(t)}\right]^{\prime} & =\left[p_{1}+q_{1} w\left(t ; p_{s}, q_{s}\right)+\delta \frac{z_{1}(t)}{m(t)}\right]\left[1-w\left(t ; p_{s}, q_{s}\right)\right] .
\end{aligned}
$$

Equation (A1.3) perfectly matches the differential equation in Guseo and Mortarino (2014, p. 308, between (A.1) and (A.2)), where $m_{c}$ is replaced by $m(t)$ and the condition $z_{s}=0$ is added in the expression for $w\left(t ; p_{s}, q_{s}\right)$. In our case, unlike in Guseo and Mortarino (2014), we are examining competition between two products that enter the market simultaneously. If we add these two conditions to the solution of the differential equation, we obtain exactly (3.3), (3.4), and (3.5).

Appendix 2. Regression model with instantaneous sales. Estimation of the parameters involved in diffusion models is usually performed through cumulative data, as described at the end of Section 3. The main reason is that the solution of the differential equations describing the mean evolutionary trajectory refers to $z(t)$ in the univariate case (or $z_{i}(t), i=1,2$, in the competitive setup). Thus, the corresponding observed data are the cumulative sales.

As an alternative approach, instantaneous data could be used as dependent variables in a regression model, provided that the specification is modified in the following manner with respect to (3.6):

$$
s_{i}(t)=z_{i}(t+0.5 ; \beta)-z_{i}(t-0.5 ; \beta)+\xi_{i}(t), \quad i=1,2,
$$

where $s_{i}(t)=v_{i}(t+1)-v_{i}(t)$ represents observed instantaneous sales (here, monthly sales) and $z_{i}(t)=z_{i}(t ; \beta)$ is defined as in (3.6).

The reason for using the difference $z_{i}(t+0.5)-z_{i}(t-0.5)$ in (A2.4) instead of the more intuitive $z_{i}(t+1)-z_{i}(t)$ is the known symmetric approximation of a function $F(x)$,

$$
F^{\prime}(x)=\frac{F(x+h)-F(x-h)}{2 h}+O\left(h^{2}\right),
$$

which cancels out the second-order derivative, $F^{\prime \prime}(x)$. This approximation has a simple form for $h=0.5$. 
TABLE A2.1

Estimation results for model (A2.4).

\begin{tabular}{lccc}
\hline & Estimate & Standard Error & $95 \%$ Confidence Interval \\
\hline$K$ & $4.9794 * 10^{7}$ & $7.9471 * 10^{5}$ & $\left(4.8231 * 10^{7}, 5.1357 * 10^{7}\right)$ \\
$p_{c}$ & $2.2006 * 10^{-3}$ & $1.9303 * 10^{-4}$ & $\left(1.8210 * 10^{-3}, 2.5801 * 10^{-3}\right)$ \\
$q_{c}$ & $4.4731 * 10^{-2}$ & $1.5710 * 10^{-3}$ & $\left(4.1641 * 10^{-2}, 4.7820 * 10^{-2}\right)$ \\
$p_{1}$ & $3.4950 * 10^{-3}$ & $2.4323 * 10^{-4}$ & $\left(3.0167 * 10^{-3}, 3.9733 * 10^{-3}\right)$ \\
$q_{1}$ & $1.4902 * 10^{-2}$ & $1.3948 * 10^{-3}$ & $\left(1.2160 * 10^{-2}, 1.7645 * 10^{-2}\right)$ \\
$p_{2}$ & $-9.3386 * 10^{-4}$ & $1.8066 * 10^{-4}$ & $\left(-1.2891 * 10^{-3},-5.7859 * 10^{-4}\right)$ \\
$q_{2}$ & $-6.2316 * 10^{-4}$ & $1.8463 * 10^{-3}$ & $\left(-4.2537 * 10^{-3}, 3.0074 * 10^{-3}\right)$ \\
$\delta$ & $-2.5405 * 10^{-2}$ & $3.5526 * 10^{-3}$ & $\left(-3.2391 * 10^{-2},-1.8419 * 10^{-2}\right)$ \\
\hline$R^{2}=0.96777$ & & \\
\hline
\end{tabular}

TABLE A2.2

Comparison between model (A2.4) and Guseo and Mortarino's (2014) model estimated with instantaneous sales.

\begin{tabular}{ccc}
\hline & $R^{2}$ & $\rho^{2}$ \\
\hline model (A2.4) & 0.9678 & 0.9678 \\
Guseo and Mortarino's (2014) model & 0.8920 & 0.8937 \\
\hline & $\widetilde{R}^{2}=0.7016$ & $F=432.57$ \\
\hline
\end{tabular}

If we use model (A2.4), we obtain the parameter estimates shown in Table A2.1. A comparison with results shown in Table 1 highlights that the procedure relying on cumulative data leads uniformly to smaller standard errors for all the parameters.

From Figure A2.1, comparing the estimated mean trajectory with model (3.6) (already shown in Figure 2) and with model (A2.4), we see that the two methods give rise to almost overlapping paths, even if the squared correlation coefficient between observed and fitted values is slightly higher when instantaneous data are used (0.9678 vs. 0.9672). Further, the estimate of the dynamic market potential structure, $\hat{m}(t)$, is fully coherent with the one obtained from model (3.6) (see Figure A2.2).

In Section 5, the comparison between the CDMP model, (3.6), and the simpler model of Guseo and Mortarino (2014) was conducted through a test statistic based upon the respective $R^{2}$ values. Of course, if we use model (A2.4) for the comparison, we should estimate Guseo and Mortarino's (2014) model with the same approach (using instantaneous sales as response variables). Table A2.2 shows the comparison. The change in the estimation method does not modify the conclusions regarding the superiority of a dynamic market potential structure.

Appendix 3. Predictive confidence bands. Starting from Srinivasan and Mason (1986) and, more recently, Boswijk and Franses (2005), we 


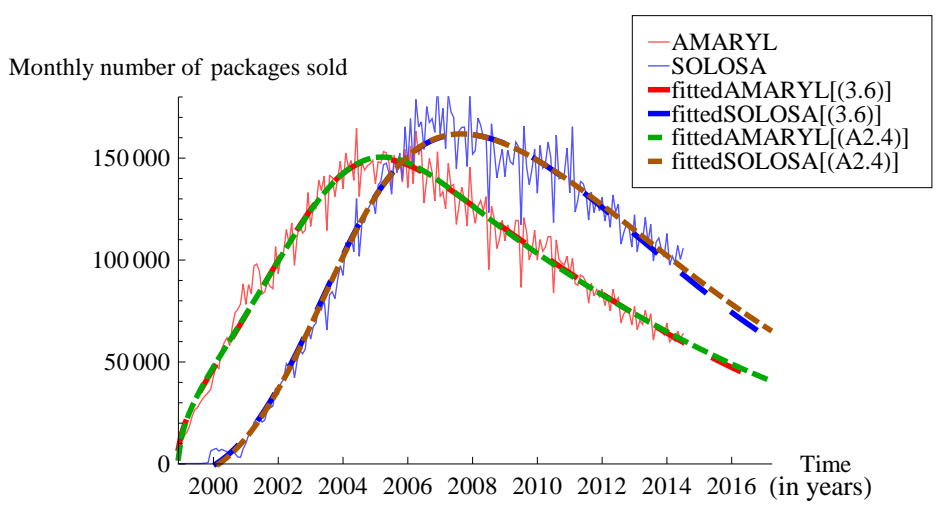

FIG A2.1. Comparison of the observed and fitted values, instantaneous sales, model (A2.4) (the fitted values with the CDMP model, (3.6), are also shown).

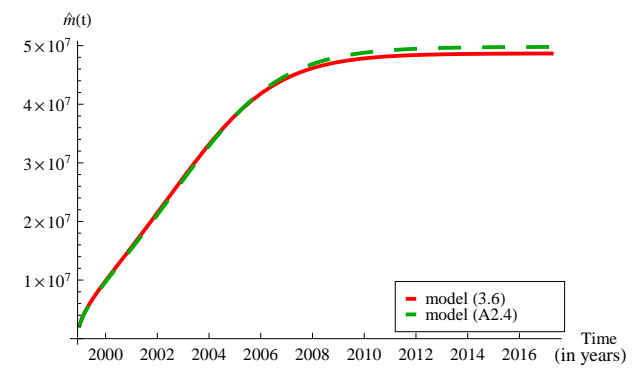

FIG A2.2. Comparison of the estimated market potential function $\hat{m}(t)$ with the CDMP, (3.6), and (A2.4) models. 

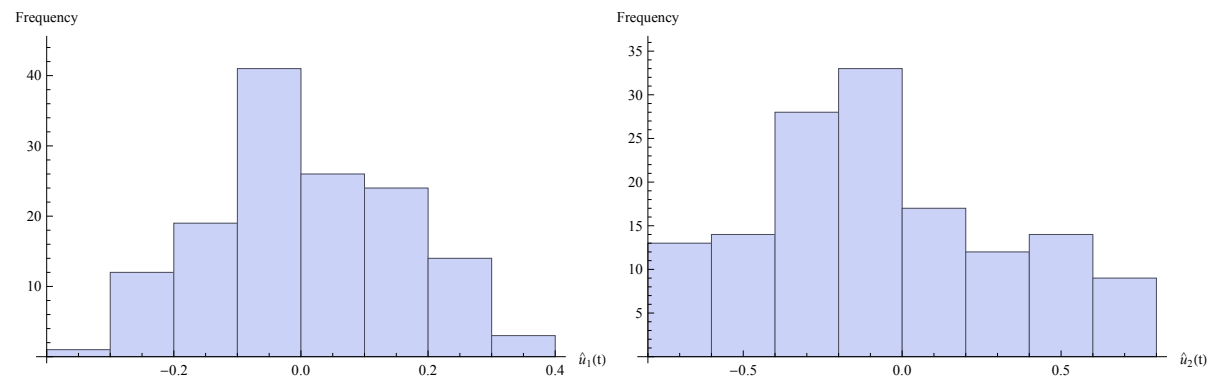

FIG A3.3. Histograms of the estimates $\hat{u}_{i}(t), i=1,2, t=49, \ldots, 188$, based on the CDMP model, (3.6).

know that - for the models under study - estimators do not have standard asymptotic properties and the parameters cannot be estimated consistently even as the sample period increases. Intuitively, since we describe finite diffusion processes, an increase in sample size with observations for $t$ values after the end of the process does not correspond to an increase in information.

The procedure used to estimate the parameters using cumulative data (model (3.6)) with a nonlinear least squares algorithm (NLS) does not at first require detailed assumptions about the structure of $\varepsilon_{i}(t)$. Following Boswijk and Franses (2005), it is, however, important to allow for heteroscedasticity in $\varepsilon_{i}(t)$. Alternative model specifications may be appropriate, but a sensible assumption is

$$
v_{i}(t)=z_{i}(t ; \beta)+\varepsilon_{i}(t)=z_{i}(t ; \beta)+z_{i}^{\prime}(t ; \beta) u_{i}(t)
$$

where $u_{i}(t)$ are supposed to be normally distributed, with zero mean and constant variance, ${ }_{u} \sigma_{i}^{2}$, for $i=1,2$. The structure (A3.5) takes into account the specific heterogeneity issue typical of saturating diffusion models (low variability around the mean trajectory both at the beginning and at the end of the diffusion cycle, with higher variability when the diffusion peaks).

The data described in this paper support the structure (A3.5), as is evident from Figure A3.3, representing the histograms of the estimates $\hat{u}_{i}(t), i=1,2, t=49, \ldots, 188$, obtained through the residuals of model (3.6). An examination of Figure 2 reveals that for both series, the fit in the first part of the series is worse and the estimate of the stochastic component, based upon residuals inflated by a partial lack-of-fit, is biased. To avoid that effect, since the purpose is to build plausible confidence bands for the future evolution of the series, we have excluded the first 48 residuals (4 years) of both series. The normality assumption is confirmed, and the corresponding 

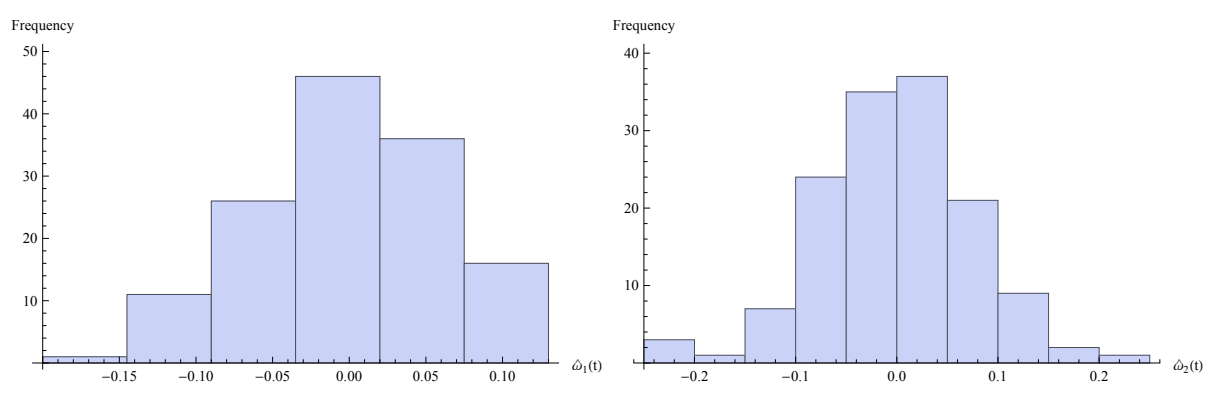

FIG A3.4. Histograms of the estimates $\hat{\omega}_{i}(t), i=1,2$ based on model (A2.4).

variance estimates are ${ }_{u} \hat{\sigma}_{1}^{2}=0.0231$ and ${ }_{u} \hat{\sigma}_{2}^{2}=0.1436$. Thus, we can use the following values as confidence bands for the predictions $\hat{z}_{i}(t)=z_{i}(t ; \hat{\beta})$, i.e.,

$$
\hat{z}_{i}(t) \pm 2_{u} \hat{\sigma}_{i} \hat{z}_{i}^{\prime}(t), \quad t=189, \ldots
$$

When the fitted values are represented in terms of instantaneous data (as in Figure A2.1), the prediction confidence bands have to be modified in the following manner. Beginning from instantaneous observed data,

$$
\begin{array}{rll}
s_{i}(t) & \stackrel{ }{=} & v_{i}(t+1)-v_{i}(t) \\
& \stackrel{(A 3.5)}{=} & z_{i}(t+1)+z_{i}^{\prime}(t+1) u_{i}(t+1)-z_{i}(t)-z_{i}^{\prime}(t) u_{i}(t) \\
& = & z_{i}(t+1)-z_{i}(t)+z_{i}^{\prime}(t+1) u_{i}(t+1)-z_{i}^{\prime}(t) u_{i}(t),
\end{array}
$$

and thus the amplitude of the confidence bands around the estimated trajectory,

$$
\hat{z}_{i}(t+1)-\hat{z}_{i}(t)
$$

should be proportional to the standard error of

$$
z_{i}^{\prime}(t+1) u_{i}(t+1)-z_{i}^{\prime}(t) u_{i}(t)
$$

given by

$$
\left.\left[\hat{z}_{i}^{\prime}(t+1)\right]^{2}+\left[\hat{z}_{i}^{\prime}(t)\right]^{2}-2 \hat{z}_{i}^{\prime}(t+1) \hat{z}_{i}^{\prime}(t)\right) \widehat{\operatorname{Cov}}\left[u_{i}(t+1), u_{i}(t)\right] .
$$

Note that the last term of the previous expression must not be neglected and equals 0.0207 for $i=1$ and 0.1419 for $i=2$. The resulting confidence bands are plotted in Figure 5 .

When we turn to the regression model (A2.4), an analogous argument leads to

(A3.6)

$s_{i}(t)=z_{i}(t+0.5)-z_{i}(t-0.5)+\xi_{i}(t)=z_{i}(t+0.5)-z_{i}(t-0.5)+z_{i}^{\prime}(t) \omega_{i}(t)$, 


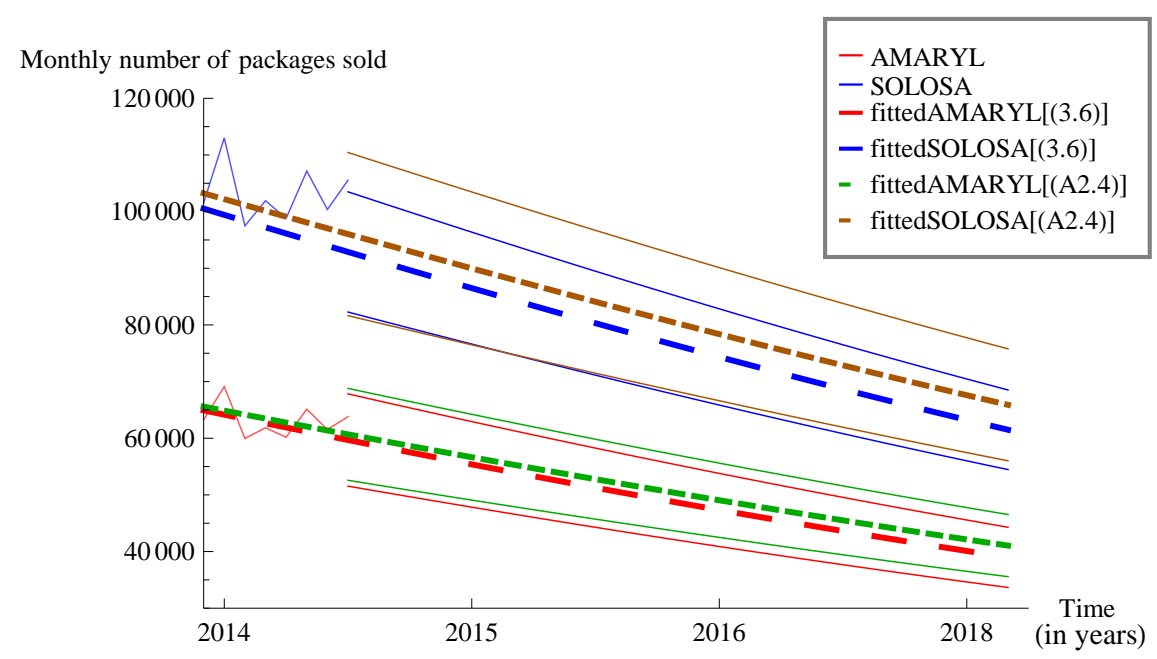

FIG A3.5. Forecasted mean sales trajectory and confidence bands, model (A2.4).

where $\omega_{i}(t)$ are supposed to be normally distributed, with zero mean and constant variance, $\omega_{\omega} \sigma_{i}^{2}, i=1,2$.

The data described in this paper support the structure (A3.5), as is evident from Figure A3.4, representing the histograms of the estimates $\hat{\omega}_{i}(t), i=1,2$, obtained through the residuals of model (A2.4). The first 48 residuals of both series were excluded for the same reason described for the residuals of model (3.6). The normality assumption is confirmed and the corresponding variance estimates are ${ }_{\omega} \hat{\sigma}_{1}^{2}=0.0045$ and ${ }_{\omega} \hat{\sigma}_{2}^{2}=$ 0.0056. Thus, we can use the following confidence bands for the predictions $\hat{z}_{i}(t+0.5)-\hat{z}_{i}(t-0.5)$, i.e.,

$$
\hat{z}_{i}(t+0.5)-\hat{z}_{i}(t-0.5) \pm 2{ }_{\omega} \hat{\sigma}_{i} \hat{z}_{i}^{\prime}(t), \quad t=189, \ldots
$$

The resulting confidence bands are plotted in Figure A3.5 together with the confidence bands obtained through model (3.6). Again, we observe that model (3.6) leads to greater precision: We obtained narrower bands for Solosa and very similar bands for Amaryl.

Appendix 4. SARMAX refinement. As mentioned in Section 4, for short-term prediction, we use a two-step procedure. First, we apply a robust NLS algorithm to model (3.6), which ignores the stochastic structure of $\varepsilon_{i}(t)$, under the well-known Levenberg-Marquardt correction of the Gauss-Newton recursive procedure (see, e.g., Seber and Wild, 2003). Second, the prediction 


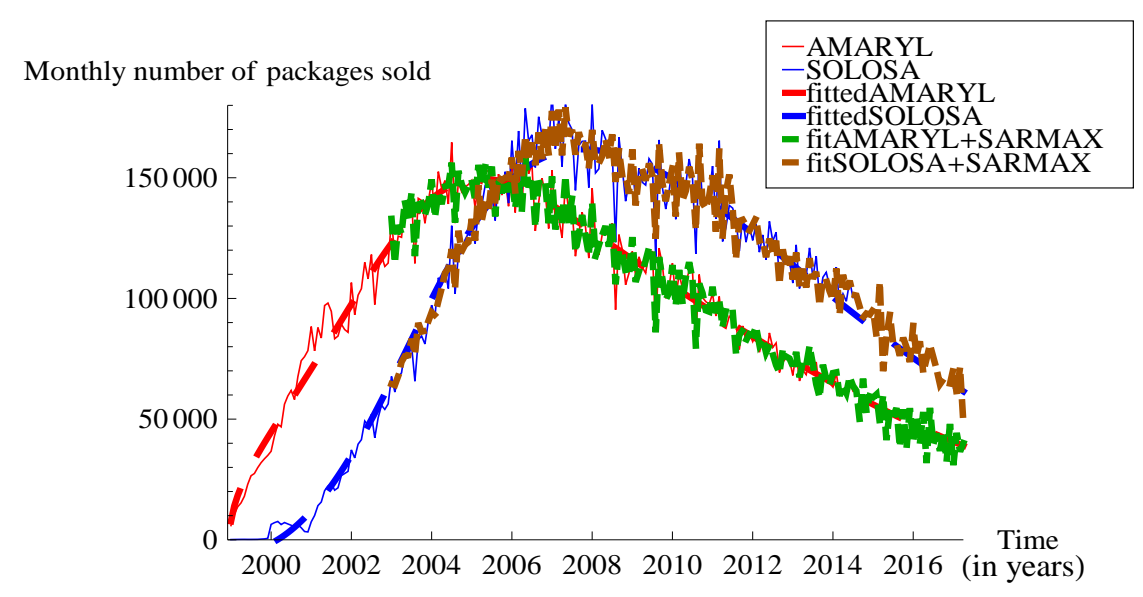

FIG A4.6. Comparison of the fitted values using the CDMP model, (3.6), and the SARMAX refinement.

$z_{i}(t ; \hat{\beta})$, based on an NLS solution, $\hat{\beta}$, may be used, as an input $\mathrm{X}$, in a model based on a seasonal, autoregressive, moving average process (SARMAX) to improve short-term prediction, which is relevant for managerial applications. This second step is implemented if the residuals of the first stage do not follow a standard white noise pattern. The Durbin-Watson statistic may be used as an exploratory test to diagnose whether this second step is necessary. In this case, the Durbin-Watson statistic equals 0.0847 , distinctly detecting a positive autocorrelation.

The SARMAX improvement for short-term predictions rests on the following equation based on a polynomial function of backward operators, namely,

$$
\Psi(B) \Phi\left(B^{s}\right)\left\{s_{i}(t)-c_{i}\left[z_{i}(t+0.5 ; \hat{\beta})-z_{i}(t-0.5 ; \hat{\beta})\right]\right\}=\Omega(B) \Theta\left(B^{s}\right) a_{i}(t)
$$

with $a_{i}(t)$ a White Noise process; $B$ and $B^{s}$ the standard and seasonal backward operators; and $\Psi(B), \Phi\left(B^{s}\right), \Omega(B)$, and $\Theta\left(B^{s}\right)$ the usual backward polynomials of order $g, G, h$, and $H$, respectively. The calibration parameters $c_{i}$ allow a global assessment of the stability of the predicted regressive values stemming from the estimated models $z_{i}(t ; \hat{\beta})$.

As above, since the first part of the series produced higher residuals and since SARMAX is meant as an improvement for predictive purposes, we 
TABle A 4.3

Parameter estimates for the SARMAX refinement. [ ] t statistic. *: significant, 95\%. **: strongly significant, 99\%. prM denotes the fitted values with the CDMP model, (3.6). The subscripts of $\tilde{R}^{2}$ and $F$ define the involved nested models; in particular, $M \mid S$ denotes the comparison between the CDMP model and the SARMAX model.

\begin{tabular}{|c|c|c|c|}
\hline Model & Parameter & Amaryl & Solosa \\
\hline \multirow{22}{*}{$\begin{array}{c}\text { SARMAX } \\
+ \\
\text { prM }\end{array}$} & AR1 & $-0.9096 * *$ & 0.1372 \\
\hline & AR2 & 0.0446 & $1.0304^{* *}$ \\
\hline & AR3 & $0.6661^{* *}$ & 0.7554 \\
\hline & AR4 & 0.2504 & -0.4155 \\
\hline & AR5 & $-0.4528 * *$ & $-0.7863^{* *}$ \\
\hline & AR6 & - & 0.2391 \\
\hline & MA1 & $-0.6233^{* *}$ & 0.2806 \\
\hline & MA2 & $0.2330 *$ & $0.8279 * *$ \\
\hline & MA3 & $0.4287^{* *}$ & 0.4580 \\
\hline & MA4 & $-0.3284 * *$ & -0.5807 \\
\hline & MA5 & $-0.7675^{* *}$ & $-0.3927 *$ \\
\hline & MA6 & - & 0.3771 \\
\hline & SAR1 & $0.6777^{* *}$ & $1.0623^{* *}$ \\
\hline & SAR2 & $-0.7824^{* *}$ & -0.0361 \\
\hline & SAR3 & $1.0223^{* *}$ & $-0.8886 * *$ \\
\hline & SAR4 & - & $1.8993^{* *}$ \\
\hline & SAR5 & - & $-0.7673^{* *}$ \\
\hline & SMA1 & 0.1788 & $0.6459^{* *}$ \\
\hline & SMA2 & $-0.8049 * *$ & $0.2042^{*}$ \\
\hline & SMA3 & $0.6395^{* *}$ & $-0.8361 * *$ \\
\hline & SMA4 & - & $1.5490^{* *}$ \\
\hline & SMA5 & - & -0.0185 \\
\hline prM & $c$ & $0.9885^{* *}$ & $1.0067 * *$ \\
\hline$\left[t_{M}\right]$ & & {$[32.4488]$} & {$[16.2051]$} \\
\hline RSS & & $2.3043 * 10^{9}$ & $9.0195 * 10^{9}$ \\
\hline $\mathrm{RSS}_{M}$ & & $8.3637 * 10^{9}$ & $1.5361 * 10^{10}$ \\
\hline$\tilde{R}_{M \mid S}^{2}$ & & 0.7245 & 0.4128 \\
\hline$F_{M \mid S}$ & & 17.8228 & 3.3985 \\
\hline
\end{tabular}


chose to apply it only to the second part of the series $(t>48)$, whose data are more relevant for future evolution.

The estimates of the parameters involved in Equation (A4.7) applied to instantaneous data are proposed in Table A4.3. The agreement between the observed data and the fitted values with the SARMAX refinement is almost perfect (see Figure A4.6). This confirms that the discrepancies between the observed data and the fitted values with the CDMP model were essentially due only to autoregressive/moving average components and seasonal effects.

Further, the overlapping of the fitted trajectory on the observed data strongly supports the choice of model (3.6) for the description of the mean trajectories of the sales data for both drugs.

\section{Appendix 5. Simulation study.}

A5.1. Correct specification. In order to assess the performance of the proposed CDMP model for different fluctuation levels around the mean trajectory, a simulation study has been performed as follows. We started from a fixed parameter configuration,

$$
\beta_{0}=\{10000,0.07,0.04,0.02,0.03,0.005,0.1,0.05\} .
$$

We simulated 1000 instantaneous datasets as

$$
s_{i}(t)=z_{i}^{\prime}\left(t ; \beta_{0}\right)+z_{i}^{\prime}\left(t ; \beta_{0}\right) \omega_{i}(t), \quad i=1,2, \quad t=1, \ldots, 50,
$$

where $\left(\omega_{1}(t), \omega_{2}(t)\right) \sim \mathcal{N}_{2}\left({ }_{\omega} \sigma_{1}^{2},{ }_{\omega} \sigma_{2}^{2},-0.1_{\omega} \sigma_{1 \omega} \sigma_{2}\right)$. The correlation value has been fixed at -0.1 to represent a substitution effect between the sales of the two products.

The ${ }_{\omega} \sigma_{i}$ were allowed to vary in the set $\{0.05,0.10,0.15,0.20,0.25\}$. As an example, Figure A5.7 shows the plot of the first simulated dataset in the case $\omega_{i} \sigma_{i}=0.05$ (a) and $\omega_{i}=0.25$ (b). The latter case corresponds to a situation with a very high noise-to-signal ratio. For each simulated dataset, the corresponding cumulative data have been used to fit the CDMP model, (3.6). Notice that this situation corresponds to a correct specification, because, for data simulation, we used the mean instantaneous trajectories, $z_{i}(t)$, with $m(t)$ specified as in (2.4).

Table A5.4 shows the mean squared error (MSE) of the parameter estimates

$$
\operatorname{MSE}\left(\hat{\beta}_{j}\right)=\frac{1}{1000} \sum_{k=1}^{1000}\left(\hat{\beta}_{j k}-\beta_{j 0}\right)^{2},
$$

where $\hat{\beta}_{j k}$ denotes the estimate of the $j$-th parameter obtained with the $k$-th simulated dataset. 


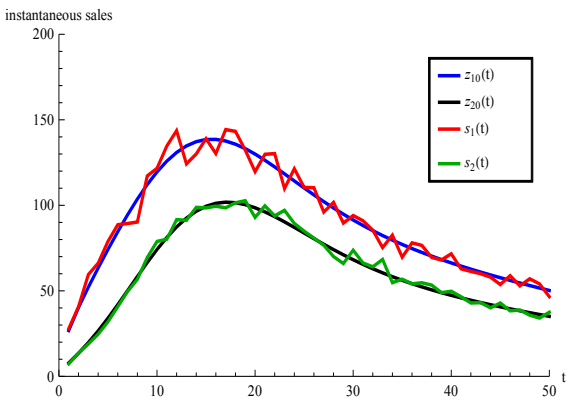

(a)

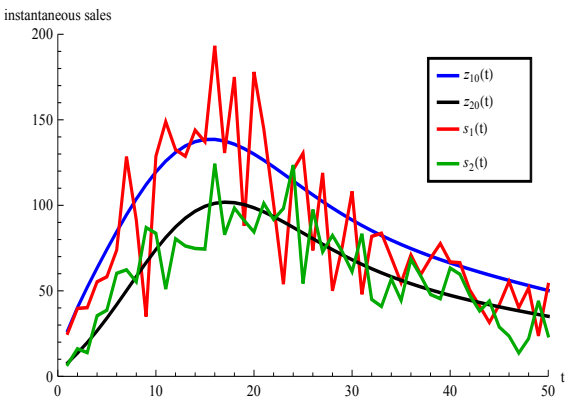

(b)

FIG A5.7. First simulated instantaneous dataset for $\omega_{\omega} \sigma_{i}=0.05$ (a) and $\omega_{\omega} \sigma_{i}=0.25$ (b).

TABLE A5.4

MSE of the parameter estimates

\begin{tabular}{ccccc}
\hline $\begin{array}{c}\text { parameter } \\
\left(\beta_{j 0}\right)\end{array}$ & $\begin{array}{c}K \\
(10000)\end{array}$ & $\begin{array}{c}p_{c} \\
(0.007)\end{array}$ & $\begin{array}{c}q_{c} \\
(0.04)\end{array}$ & $\begin{array}{c}p_{1} \\
(0.02)\end{array}$ \\
\hline${ }_{\omega} \sigma_{i}=0.05$ & $7.0655^{*} 10^{5}$ & $1.5130^{*} 10^{-7}$ & $9.1726^{*} 10^{-5}$ & $1.1897^{*} 10^{-5}$ \\
$\omega \sigma_{i}=0.10$ & $7.7058^{*} 10^{5}$ & $3.8166^{*} 10^{-7}$ & $1.3603^{*} 10^{-4}$ & $2.5986^{*} 10^{-5}$ \\
$\omega \sigma_{i}=0.15$ & $1.2554^{*} 10^{6}$ & $8.3944^{*} 10^{-7}$ & $2.3944^{*} 10^{-4}$ & $5.5103^{*} 10^{-5}$ \\
$\omega \sigma_{i}=0.20$ & $4.6040^{*} 10^{7}$ & $4.2749^{*} 10^{-6}$ & $5.0578^{*} 10^{-4}$ & $6.7359^{*} 10^{-5}$ \\
$\omega \sigma_{i}=0.25$ & $5.4571^{*} 10^{8}$ & $7.7279^{*} 10^{-6}$ & $8.2287^{*} 10^{-4}$ & $3.4129^{*} 10^{-4}$ \\
\hline \multicolumn{1}{c}{} & & & & \\
parameter & $q_{1}$ & $p_{2}$ & $q_{2}$ & $\delta$ \\
$\left(\beta_{j 0}\right)$ & $(0.03)$ & $(0.005)$ & $(0.1)$ & $(0.05)$ \\
\hline${ }_{\omega} \sigma_{i}=0.05$ & $2.7558^{*} 10^{-3}$ & $1.4823^{*} 10^{-6}$ & $3.5251^{*} 10^{-3}$ & $8.9532^{*} 10^{-3}$ \\
$\omega \sigma_{i}=0.10$ & $8.3951^{*} 10^{-3}$ & $6.5940^{*} 10^{-6}$ & $9.1042^{*} 10^{-3}$ & $2.4722^{*} 10^{-2}$ \\
$\omega \sigma_{i}=0.15$ & $3.1755^{*} 10^{-2}$ & $2.1710^{*} 10^{-5}$ & $3.2648^{*} 10^{-2}$ & $9.0742^{*} 10^{-2}$ \\
$\omega \sigma_{i}=0.20$ & $6.7459^{*} 10^{-2}$ & $3.1218^{*} 10^{-5}$ & $6.9825^{*} 10^{-2}$ & $1.8678^{*} 10^{-1}$ \\
$\omega \sigma_{i}=0.25$ & $9.3869^{*} 10^{-1}$ & $2.7502^{*} 10^{-4}$ & $9.4350^{*} 10^{-1}$ & 2.6786 \\
\hline
\end{tabular}


TABLE A5.5

MISE for the market potential function, $m(t)$

\begin{tabular}{cccccc}
\hline & $T=50$ & $T=60$ & $T=70$ & $T=88$ & $T=114$ \\
\hline${ }_{\omega} \sigma_{i}=0.05$ & $7.9842^{*} 10^{5}$ & $8.4136^{*} 10^{5}$ & $9.5680^{*} 10^{5}$ & $1.8808^{*} 10^{6}$ & $6.7524^{*} 10^{6}$ \\
$\omega \sigma_{i}=0.10$ & $1.0264^{*} 10^{6}$ & $1.1166^{*} 10^{6}$ & $1.3408^{*} 10^{6}$ & $2.8253^{*} 10^{6}$ & $9.2680^{*} 10^{6}$ \\
${ }_{\omega} \sigma_{i}=0.15$ & $1.6529^{*} 10^{6}$ & $1.8306^{*} 10^{6}$ & $2.2428^{*} 10^{6}$ & $4.7968^{*} 10^{6}$ & $1.5172^{*} 10^{7}$ \\
$\omega \sigma_{i}=0.20$ & $3.8034^{*} 10^{6}$ & $4.1396^{*} 10^{6}$ & $4.8721^{*} 10^{6}$ & $9.0723^{*} 10^{6}$ & $2.7517^{*} 10^{7}$ \\
${ }_{\omega} \sigma_{i}=0.25$ & $6.3988^{*} 10^{7}$ & $6.9203^{*} 10^{7}$ & $8.0086^{*} 10^{7}$ & $1.3918^{*} 10^{7}$ & $3.8715^{*} 10^{7}$ \\
\hline
\end{tabular}

For ${ }_{\omega} \sigma_{i} \leq 0.20$, we observe a gradual deterioration in the accuracy of the estimates of the evolutionary parameters $\left(p_{1}, q_{1}, p_{2}, q_{2}\right.$, and $\left.\delta\right)$. The estimates are quite unstable in the case $\omega_{i}=0.25$, corresponding to an extreme value of the noise-to-signal ratio. Notice that the MSE, as an average, may be heavily affected by some odd convergence points for a few simulated datasets. Due to the high number of datasets, the estimation procedure is unsupervised and common initial values have been used for all of them.

One interesting issue is to see whether the model allows for good estimates of the market potential component, $m(t)$. Thus the mean integrated squared error (MISE) has been evaluated as follows:

$$
\operatorname{MISE}(\hat{m}(t))=\frac{1}{1000} \sum_{k=1}^{1000} \int_{t=0}^{T}\left[\hat{m}_{k}(t)-m_{0}(t)\right]^{2} d t
$$

where $\hat{m}_{k}(t)$ denotes the estimate of $m(t)$ obtained with the $k$-th simulated dataset, $m_{0}(t)$ denotes the true function $m(t)$ used to generate the data, and $T=50,60,70,88,114$. The two final values represent, respectively, the 95 -th and 99-th quantiles of $z_{i}\left(t ; \beta_{0}\right)$. In other words, after 114 , the products' lifecycle is essentially concluded and the estimation issue is no longer interesting (firms usually stop offering a product when sales levels are negligible). Because a product's exit from the market is often anticipated due to high commercialization costs, the 95-th quantile, 88, is also an interesting endpoint to consider. Notice that the simulated data used to fit the model cover the first 50 time points. This is why the first $T$ value has been set at 50 . The values 60 and 70 represent, for forecasting purposes, a medium term and a long term. Table A5.5 shows the MISE values. Unlike the MSE values, the MISE results do not highlight a sudden jump for high ${ }_{\omega} \sigma_{i}$ values, but they smoothly increase with $\omega_{i}$ values.

The left side of Figures A5.8-A5.12 shows the plots of $m_{0}(t), \hat{m}_{k}(t)$, $\bar{m}(t)=\frac{1}{1000} \sum_{k=1}^{1000} \hat{m}_{k}(t)$, the median trajectory, and the quantile trajectories (0.05 and 0.95). Because our main interest is in the global model function, however, the right side of Figures A5.8-A5.12 shows the plots 

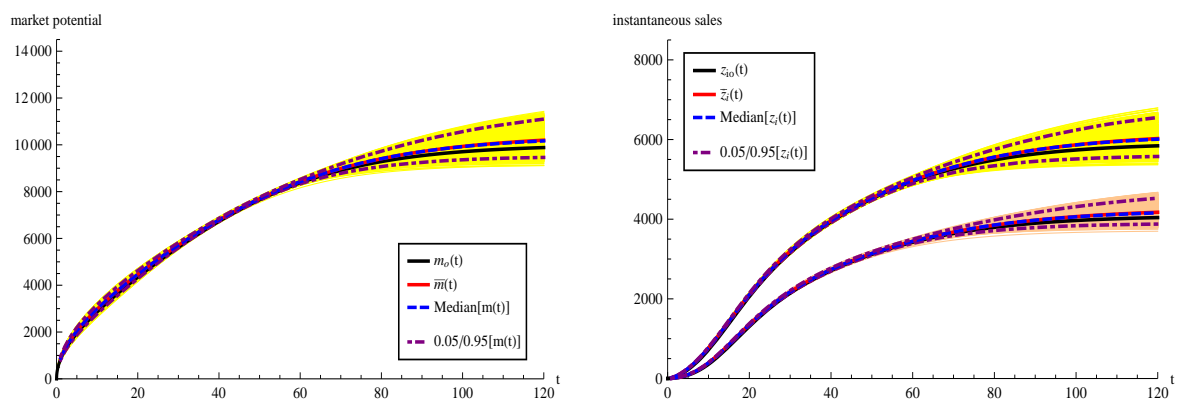

Fig A5.8. Case $\omega_{i}=0.05$. Left side: $\hat{m}_{k}(t)$ (yellow trajectories), true $m_{0}(t), \bar{m}(t)=$ $\frac{1}{1000} \sum_{k=1}^{1000} \hat{m}_{k}(t)$, median trajectory, and quantile trajectories (0.05 and 0.95). Right side: $\hat{z}_{i k}(t)$ (yellow and orange trajectories), true $z_{i 0}(t), \bar{z}_{i}(t)=\frac{1}{1000} \sum_{k=1}^{1000} \hat{z}_{i k}(t)$, median trajectory, and quantile trajectories (0.05 and 0.95).
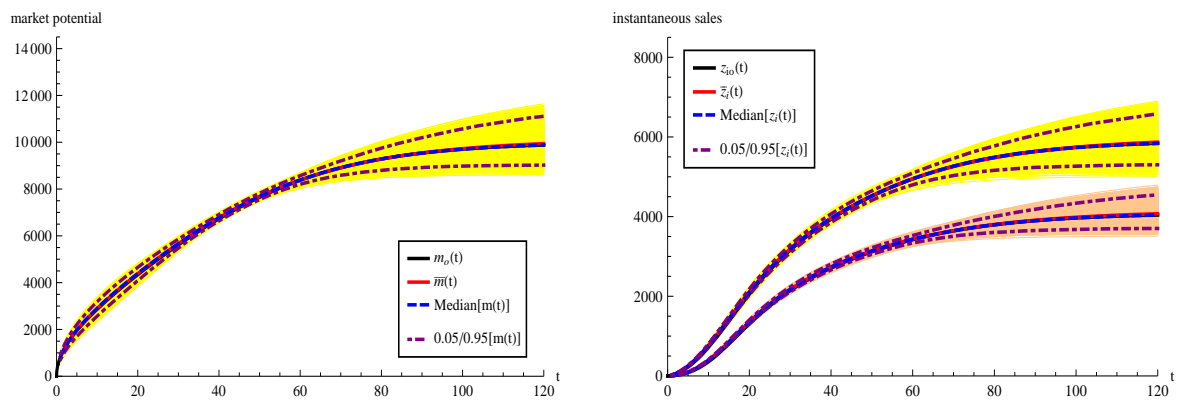

FIG A5.9. Case ${ }_{\omega} \sigma_{i}=0.10$. Left side: $\hat{m}_{k}(t)$ (yellow trajectories), true $m_{0}(t), \bar{m}(t)=$ $\frac{1}{1000} \sum_{k=1}^{1000} \hat{m}_{k}(t)$, median trajectory, and quantile trajectories (0.05 and 0.95). Right side: $\hat{z}_{i k}(t)$ (yellow and orange trajectories), true $z_{i 0}(t), \bar{z}_{i}(t)=\frac{1}{1000} \sum_{k=1}^{1000} \hat{z}_{i k}(t)$, median trajectory, and quantile trajectories (0.05 and 0.95).
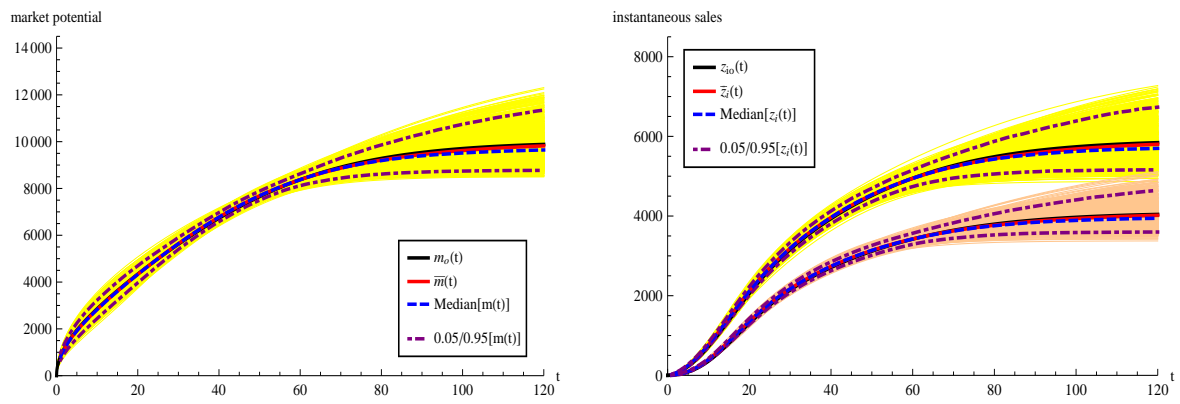

FIG A5.10. Case ${ }_{\omega} \sigma_{i}=0.15$. Left side: $\hat{m}_{k}(t)$ (yellow trajectories), true $m_{0}(t), \bar{m}(t)=$ $\frac{1}{1000} \sum_{k=1}^{1000} \hat{m}_{k}(t)$, median trajectory, and quantile trajectories (0.05 and 0.95). Right side: $\hat{z}_{i k}(t)$ (yellow and orange trajectories), true $z_{i 0}(t), \bar{z}_{i}(t)=\frac{1}{1000} \sum_{k=1}^{1000} \hat{z}_{i k}(t)$, median trajectory, and quantile trajectories (0.05 and 0.95). 

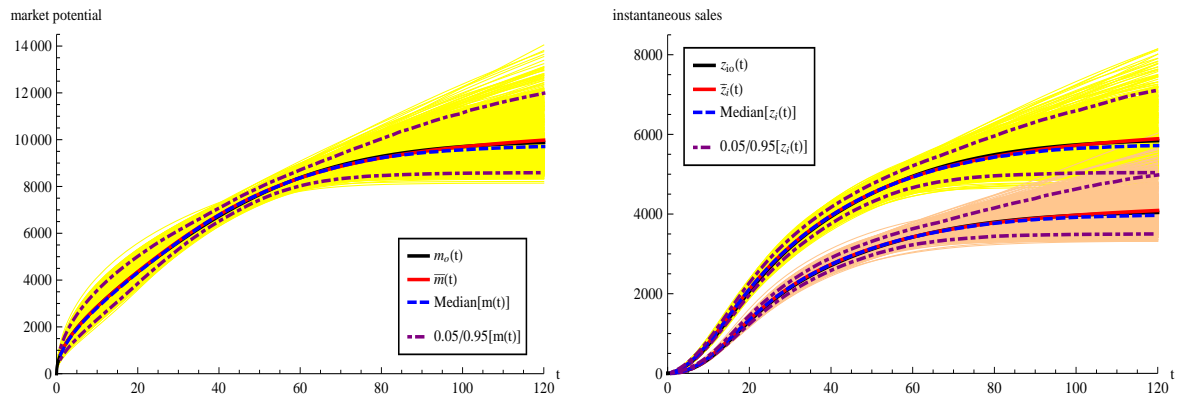

FIG A5.11. Case $\omega_{\omega} \sigma_{i}=0.20$. Left side: $\hat{m}_{k}(t)$ (yellow trajectories), true $m_{0}(t), \bar{m}(t)=$ $\frac{1}{1000} \sum_{k=1}^{1000} \hat{m}_{k}(t)$, median trajectory, and quantile trajectories (0.05 and 0.95). Right side: $\hat{z}_{i k}(t)$ (yellow and orange trajectories), true $z_{i 0}(t), \bar{z}_{i}(t)=\frac{1}{1000} \sum_{k=1}^{1000} \hat{z}_{i k}(t)$, median trajectory, and quantile trajectories (0.05 and 0.95).
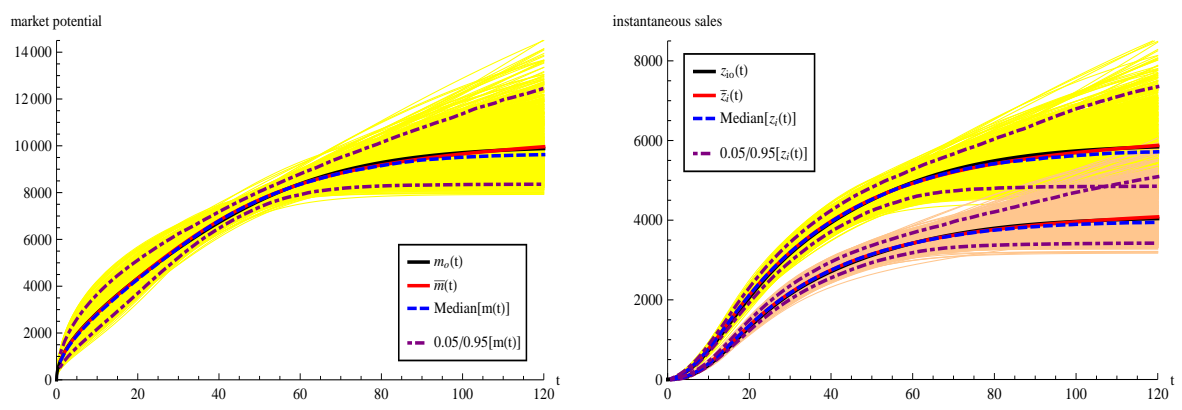

FIG A5.12. Case $\omega_{\omega} \sigma_{i}=0.25$. Left side: $\hat{m}_{k}(t)$ (yellow trajectories), true $m_{0}(t), \bar{m}(t)=$ $\frac{1}{1000} \sum_{k=1}^{1000} \hat{m}_{k}(t)$, median trajectory, and quantile trajectories (0.05 and 0.95). Right side: $\hat{z}_{i k}(t)$ (yellow and orange trajectories), true $z_{i 0}(t), \bar{z}_{i}(t)=\frac{1}{1000} \sum_{k=1}^{1000} \hat{z}_{i k}(t)$, median trajectory, and quantile trajectories (0.05 and 0.95). 
of the true $z_{i}(t)$ functions, the estimated mean trajectories $\hat{z}_{i k}(t), \bar{z}_{i}(t)=$ $\frac{1}{1000} \sum_{k=1}^{1000} \hat{z}_{i k}(t)$, the median trajectory, and the quantile trajectories $(0.05$ and 0.95$)$. For $\omega_{\omega} \sigma_{i}$ values up to 0.15 , all the estimated trajectories are very close to the true one even for long-term forecasting $(t \leq 75)$. For $\omega_{\omega} \sigma_{i}=0.20$, uncertainty is moderate for medium-term forecasting $(t \leq 60)$, while fluctuations around the true trajectories make forecasts less reliable for ${ }_{\omega} \sigma_{i}$ exceeding 0.20. It is notable, however, that the average trajectories, $\bar{m}(t)$ and $\bar{z}_{i}(t)$, are essentially coincident with the respective true functions, $m_{0}(t)$ and $z_{0 i}(t)$.

A5.2. Alternative market potential structures. The simulations shed light on one further key point. The described results were obtained with a "correctly specified" $m(t)$ function, function (2.4). To determine whether the proposed model could also adequately describe data generated with a more complex dynamic than (2.4) - and what implications such a misspecification in the market potential dynamics would have for evolutionary parameters $p_{1}$, $q_{1}, p_{2}, q_{2}$, and $\delta$-we examined alternative $m(t)$ functions for data generation. The structure (2.4) represents a communication network's size growing according to a simple Bass model. Pertaining sensible assumptions about knowledge spread may lead to heterogeneous behavior of involved agents. In the literature, this effect has been modeled through more complex diffusion of innovation models either with a continuous approach (see, e.g., Bemmaor, 1994; Bemmaor and Lee, 2002) or with a discrete approach (see, e.g., Karmeshu and Goswami, 2001). The former leads to the alternative specification

$$
m(t)=K \frac{\left[1-e^{\left.-\left(p_{c}+q_{c}\right) t\right]^{\beta}}\right.}{\left[1+\frac{q_{c}}{p_{c}} e^{-\left(p_{c}+q_{c}\right) t}\right]^{\alpha}}, \quad K, p_{c}, q_{c}, \alpha, \beta>0, \quad t>0,
$$

while the latter gives rise to a two-wave model,

$$
m(t)=K_{1} \frac{1-e^{-\left(p_{1 c}+q_{1 c}\right) t}}{1+\frac{q_{1 c}}{p_{1 c}} e^{-\left(p_{1 c}+q_{1 c}\right) t}}+K_{2} \frac{1-e^{-\left(p_{2 c}+q_{2 c}\right)\left(t-t_{c}\right)}}{1+\frac{q_{2 c}}{p_{2 c}} e^{-\left(p_{2 c}+q_{2 c}\right)\left(t-t_{c}\right)}} I_{t \geq t c},
$$

where $K_{i}, p_{i c}, q_{i c}, t_{c}>0, t>0$.

Specifically, we present the results obtained for

- a Bemmaor model (A5.8) with $\alpha=0.25$ and $\beta=0.5(\mathrm{BE}(0.25,0.5))$,

- a Bemmaor model (A5.8) with $\alpha=1$ and $\beta=0.5(\mathrm{BE}(1,0.5))$,

- a two-wave model (A5.9) with changepoint in $t c_{c}=20(\mathrm{TW}(20))$.

Notice that function (2.4) can be represented as $\mathrm{BE}(0.5,0.5)$, as it is obviously a special case of (A5.8). 


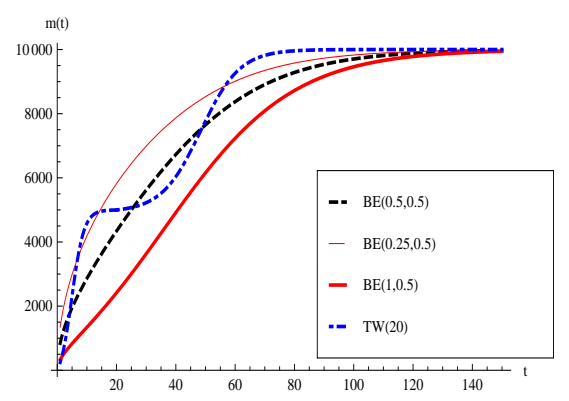

FIG A5.13. Alternative $m(t)$ dynamics used in simulations: a Bemmaor model (A5.8) with $\alpha=0.25$ and $\beta=0.5$ (BE(0.25,0.5)), a Bemmaor model (A5.8) with $\alpha=1$ and $\beta=0.5$ (BE(1,0.5)) a two-wave model (A5.9) with changepoint in $t c_{c}=20$ (TW(20)). The $m(t)$ function used throughout the paper, (2.4), is also plotted and denoted by BE(0.5,0.5).

TABLE A5.6

MISE for the market potential function, $m(t)$, with alternative specifications.

\begin{tabular}{|c|c|c|c|c|c|c|}
\hline & & $T=50$ & $T=60$ & $T=70$ & $T=88$ & $T=114$ \\
\hline \multirow[t]{3}{*}{$\mathrm{BE}(0.5,0.5)$} & $\omega \sigma_{i}=0.05$ & $7.9842^{*} 10^{5}$ & $8.4136^{*} 10^{5}$ & $9.5680^{*} 10^{5}$ & $1.8808^{*} 10^{6}$ & $6.7524^{*} 10^{6}$ \\
\hline & ${ }_{\omega} \sigma_{i}=0.10$ & $1.0264^{*} 10^{6}$ & $1.1166 * 10^{6}$ & $1.3408^{*} 10^{6}$ & $2.8253^{*} 10^{6}$ & $9.2680^{*} 10^{6}$ \\
\hline & ${ }_{\omega} \sigma_{i}=0.15$ & $1.6529 * 10^{6}$ & $1.8306 * 10^{6}$ & $2.2428^{*} 10^{6}$ & $4.7968 * 10^{6}$ & $1.5172^{*} 10^{7}$ \\
\hline \multirow[t]{3}{*}{$\mathrm{BE}(0.25,0.5)$} & $\omega \sigma_{i}=0.05$ & $1.0649^{*} 10^{6}$ & $1.1039 * 10^{6}$ & $1.2103^{*} 10^{6}$ & $2.1663 * 10^{6}$ & $8.4540^{*} 10^{6}$ \\
\hline & $\omega \sigma_{i}=0.10$ & $2.0646^{*} 10^{6}$ & $2.1692^{*} 10^{6}$ & $2.3584^{*} 10^{6}$ & $3.4547^{*} 10^{6}$ & $9.2892^{*} 10^{6}$ \\
\hline & $\omega \sigma_{i}=0.15$ & $3.9747^{*} 10^{6}$ & $4.1821^{*} 10^{6}$ & $4.5231^{*} 10^{6}$ & $6.1057^{*} 10^{6}$ & $1.3061 * 10^{7}$ \\
\hline \multirow[t]{3}{*}{$\mathrm{BE}(1,0.5)$} & $\omega \sigma_{i}=0.05$ & $1.4031^{*} 10^{6}$ & $1.4457^{*} 10^{6}$ & $1.6207^{*} 10^{6}$ & $4.1432^{*} 10^{6}$ & $1.7707^{*} 10^{7}$ \\
\hline & ${ }_{\omega} \sigma_{i}=0.10$ & $2.7012^{*} 10^{6}$ & $2.8158^{*} 10^{6}$ & $3.4295^{*} 10^{6}$ & $1.0847^{*} 10^{7}$ & $6.0820^{*} 10^{7}$ \\
\hline & $\omega \sigma_{i}=0.15$ & $3.2451 * 10^{6}$ & $3.4712^{*} 10^{6}$ & $4.5917^{*} 10^{6}$ & $1.7575^{*} 10^{7}$ & $1.1643 * 10^{8}$ \\
\hline \multirow[t]{3}{*}{$\mathrm{TW}(20)$} & $\omega \sigma_{i}=0.05$ & $5.2213^{*} 10^{7}$ & $5.4527^{*} 10^{7}$ & $5.5356^{*} 10^{7}$ & $1.3536^{*} 10^{8}$ & $1.2534^{*} 10^{9}$ \\
\hline & $\omega \sigma_{i}=0.10$ & $5.2346^{*} 10^{7}$ & $5.4871^{*} 10^{7}$ & $5.5799^{*} 10^{7}$ & $1.3644^{*} 10^{8}$ & $1.2625^{*} 10^{9}$ \\
\hline & $\omega \sigma_{i}=0.15$ & $5.3285^{*} 10^{7}$ & $5.5762 * 10^{7}$ & $5.6861^{*} 10^{7}$ & $1.4162^{*} 10^{8}$ & $1.2932^{*} 10^{9}$ \\
\hline
\end{tabular}

Figure A5.13 shows the plot of these alternative dynamic structures in comparison with the original function used in previously described simulations. For comparative purposes, we show here the results obtained with the three alternatives for the less extreme values of $\omega_{i} \sigma_{i}$ (namely $0.05,0.10$, and $0.15)$.

Table A5.6 shows the MISE values. When a $\mathrm{BE}(0.25,0.5)$ structure is used to simulate the data, the MISE values are remarkably close to the $\mathrm{BE}(0.5,0.5)$ case, and for higher $T$ values, the MISE is even smaller. The $\mathrm{BE}(1,0.5)$ model makes a somewhat greater impact on the MISE. Figures A5.14-A5.19 show the true and estimated trajectories for the dynamic market potential and the fitted response, when $m(t)$ is simulated through a Bemmaor process. The fluctuations are higher, especially when $\mathrm{BE}(1.0 .5)$ is 

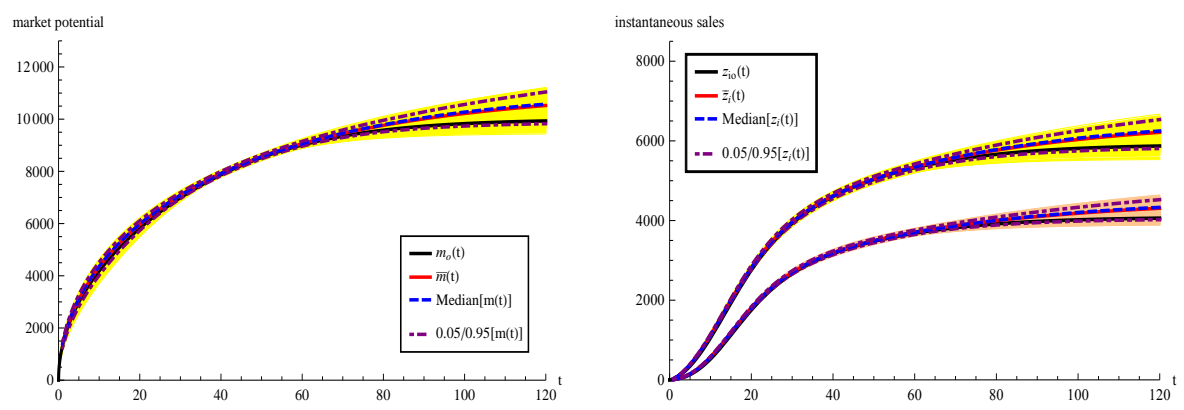

FIG A5.14. Case BE(0.25,0.5), $\omega_{\omega} \sigma_{i}=0.05$. Left side: $\hat{m}_{k}(t)$ (yellow trajectories), true $m_{0}(t), \bar{m}(t)=\frac{1}{1000} \sum_{k=1}^{1000} \hat{m}_{k}(t)$, median trajectory, and quantile trajectories $(0.05$ and 0.95). Right side: $\hat{z}_{i k}(t)$ (yellow and orange trajectories), true $z_{i 0}(t), \bar{z}_{i}(t)=$ $\frac{1}{1000} \sum_{k=1}^{1000} \hat{z}_{i k}(t)$, median trajectory, and quantile trajectories (0.05 and 0.95).
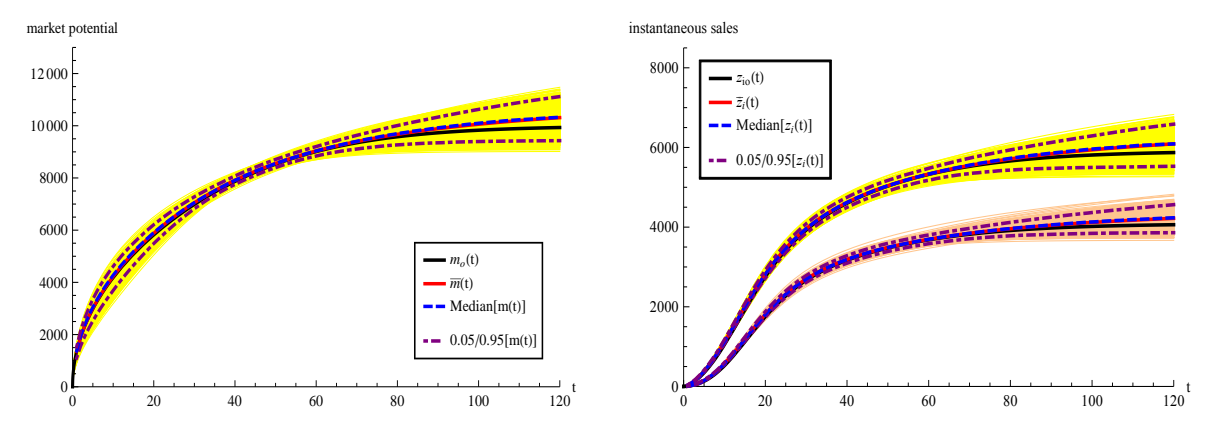

FIG A5.15. Case BE(0.25,0.5), $\omega_{i}=0.10$. Left side: $\hat{m}_{k}(t)$ (yellow trajectories), true $m_{0}(t), \bar{m}(t)=\frac{1}{1000} \sum_{k=1}^{1000} \hat{m}_{k}(t)$, median trajectory, and quantile trajectories $(0.05$ and 0.95). Right side: $\hat{z}_{i k}(t)$ (yellow and orange trajectories), true $z_{i 0}(t), \bar{z}_{i}(t)=$ $\frac{1}{1000} \sum_{k=1}^{1000} \hat{z}_{i k}(t)$, median trajectory, and quantile trajectories (0.05 and 0.95).

used and $t \geq 80$. For higher $t$ values, the average trajectories are not fully coincident with the true ones. This is unsurprising, as the estimation procedure makes use of data from $t=1$ to $t=50$; a good approximation of a Bemmaor structure through a Bass model in the range $[1,50]$ may not be equally good in a different range.

Finally, the two-wave model $(\mathrm{TW}(20))$ produces MISE values that are ten times greater than the $\mathrm{BE}(0.5,0.5)$ model. Figures A5.20-A5.22 show the true and estimated trajectories for dynamic market potential and the fitted response when $m(t)$ is simulated through a two-wave process. In this case, $m(t)$ cannot be adequately described even for smaller $t$ values, but the fluctuations around $z_{0 i}(t)$ are extremely small for $t \leq 60$.

Table A5.7 shows the MSE for the estimates of $\left(p_{1}, q_{1}, p_{2}, q_{2}, \delta\right)$ with the 

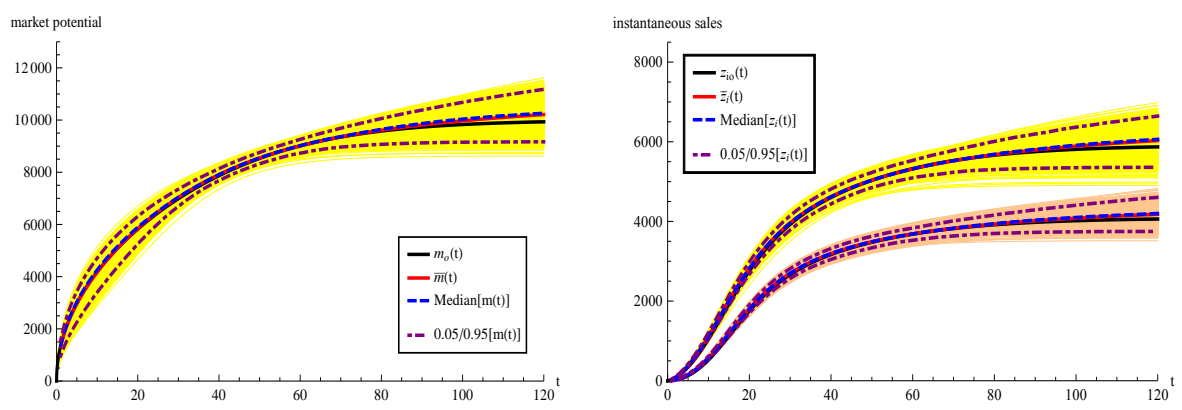

FIG A5.16. Case BE(0.25,0.5), $\omega_{\omega} \sigma_{i}=0.15$. Left side: $\hat{m}_{k}(t)$ (yellow trajectories), true $m_{0}(t), \bar{m}(t)=\frac{1}{1000} \sum_{k=1}^{1000} \hat{m}_{k}(t)$, median trajectory, and quantile trajectories $(0.05$ and 0.95). Right side: $\hat{z}_{i k}(t)$ (yellow and orange trajectories), true $z_{i 0}(t), \bar{z}_{i}(t)=$ $\frac{1}{1000} \sum_{k=1}^{1000} \hat{z}_{i k}(t)$, median trajectory, and quantile trajectories (0.05 and 0.95).
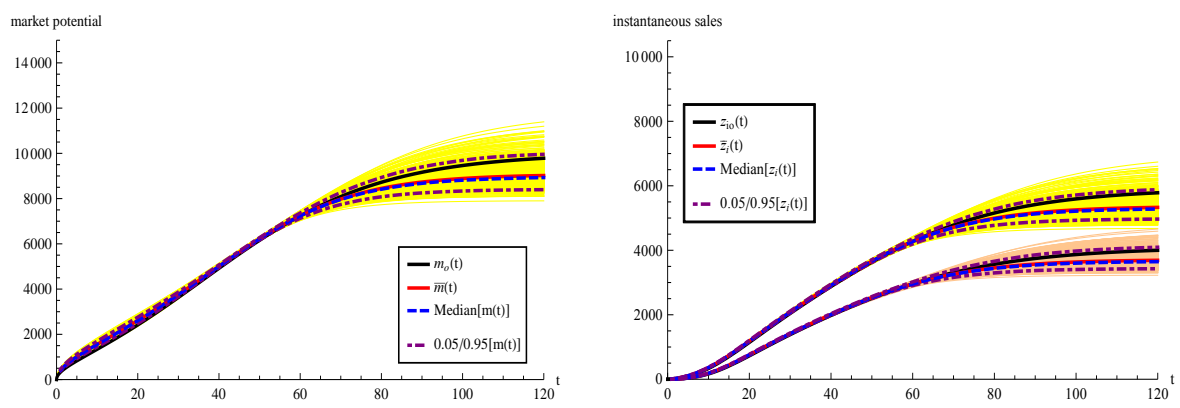

FIG A5.17. Case BE(1,0.5), $\omega_{i}=0.05$. Left side: $\hat{m}_{k}(t)$ (yellow trajectories), true $m_{0}(t)$, $\bar{m}(t)=\frac{1}{1000} \sum_{k=1}^{1000} \hat{m}_{k}(t)$, median trajectory, and quantile trajectories (0.05 and 0.95). Right side: $\hat{z}_{i k}(t)$ (yellow and orange trajectories), true $z_{i 0}(t), \bar{z}_{i}(t)=\frac{1}{1000} \sum_{k=1}^{1000} \hat{z}_{i k}(t)$, median trajectory, and quantile trajectories (0.05 and 0.95).
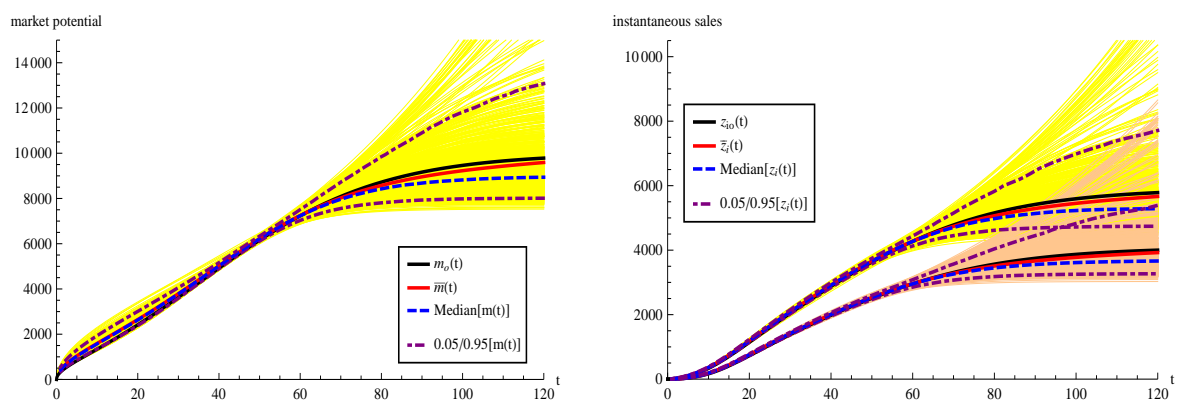

FIG A5.18. Case BE(1,0.5), $\omega_{i}=0.10$. Left side: $\hat{m}_{k}(t)$ (yellow trajectories), true $m_{0}(t)$, $\bar{m}(t)=\frac{1}{1000} \sum_{k=1}^{1000} \hat{m}_{k}(t)$, median trajectory, and quantile trajectories (0.05 and 0.95). Right side: $\hat{z}_{i k}(t)$ (yellow and orange trajectories), true $z_{i 0}(t), \bar{z}_{i}(t)=\frac{1}{1000} \sum_{k=1}^{1000} \hat{z}_{i k}(t)$, median trajectory, and quantile trajectories (0.05 and 0.95). 

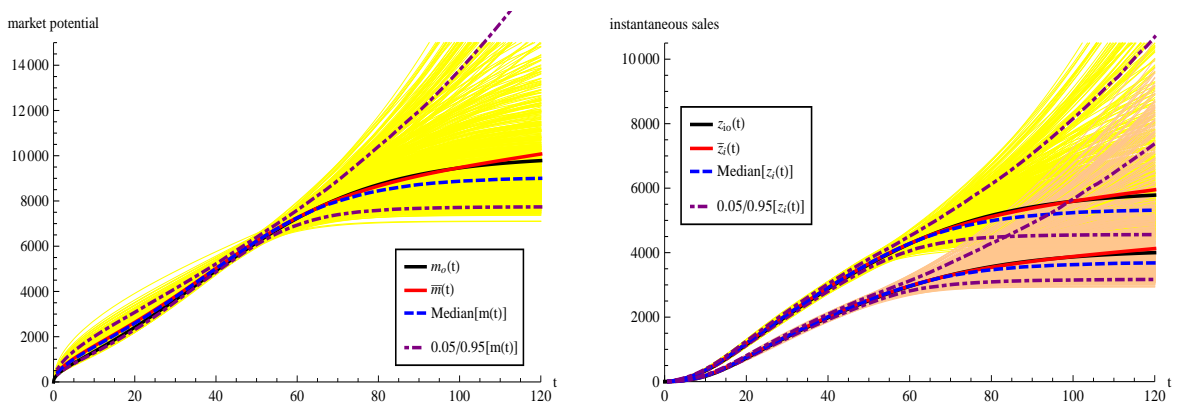

FIG A5.19. Case BE(1,0.5), ${ }_{\omega} \sigma_{i}=0.15$. Left side: $\hat{m}_{k}(t)$ (yellow trajectories), true $m_{0}(t)$, $\bar{m}(t)=\frac{1}{1000} \sum_{k=1}^{1000} \hat{m}_{k}(t)$, median trajectory, and quantile trajectories (0.05 and 0.95). Right side: $\hat{z}_{i k}(t)$ (yellow and orange trajectories), true $z_{i 0}(t), \bar{z}_{i}(t)=\frac{1}{1000} \sum_{k=1}^{1000} \hat{z}_{i k}(t)$, median trajectory, and quantile trajectories (0.05 and 0.95).

TABLE A5.7

MSE of the parameter estimates for $\left(p_{1}, q_{1}, p_{2}, q_{2}, \delta\right)$ with alternative $m(t)$ specifications.

\begin{tabular}{|c|c|c|c|c|c|c|}
\hline & $\begin{array}{l}\text { parameter } \\
\qquad\left(\beta_{j 0}\right)\end{array}$ & $\begin{array}{c}p_{1} \\
(0.02)\end{array}$ & $\begin{array}{c}q_{1} \\
(0.03)\end{array}$ & $\begin{array}{c}p_{2} \\
(0.005)\end{array}$ & $\begin{array}{c}q_{2} \\
(0.1)\end{array}$ & $\begin{array}{c}\delta \\
(0.05)\end{array}$ \\
\hline \multirow[t]{3}{*}{$\mathrm{BE}(0.5,0.5)$} & ${ }_{\omega} \sigma_{i}=0.05$ & $1.1897^{*} 10^{-5}$ & $2.7558^{*} 10^{-3}$ & $1.4823^{*} 10^{-6}$ & $3.5251^{*} 10^{-3}$ & $8.9532^{*} 10^{-3}$ \\
\hline & ${ }_{\omega} \sigma_{i}=0.10$ & $2.5986^{*} 10^{-5}$ & $8.3951 * 10^{-3}$ & $6.5940^{*} 10^{-6}$ & $9.1042^{*} 10^{-3}$ & $2.4722^{*} 10^{-2}$ \\
\hline & ${ }_{\omega} \sigma_{i}=0.15$ & $5.5103^{*} 10^{-5}$ & $3.1755^{*} 10^{-2}$ & $2.1710^{*} 10^{-5}$ & $3.2648^{*} 10^{-2}$ & $9.0742^{*} 10^{-2}$ \\
\hline \multirow[t]{3}{*}{$\mathrm{BE}(0.25,0.5)$} & $\omega \sigma_{i}=0.05$ & $1.1852^{*} 10^{-5}$ & $1.8281^{*} 10^{-3}$ & $1.1023^{*} 10^{-6}$ & $2.5001^{*} 10^{-3}$ & $6.1818^{*} 10^{-3}$ \\
\hline & $\omega \sigma_{i}=0.10$ & $2.7446^{*} 10^{-5}$ & $6.6698^{*} 10^{-3}$ & $5.0748^{*} 10^{-6}$ & $7.4257^{*} 10^{-3}$ & $1.9925^{*} 10^{-2}$ \\
\hline & ${ }_{\omega} \sigma_{i}=0.15$ & $4.9248^{*} 10^{-5}$ & $2.1805^{*} 10^{-2}$ & $1.3392 * 10^{-5}$ & $2.3126^{*} 10^{-2}$ & $6.1728^{*} 10^{-2}$ \\
\hline \multirow[t]{3}{*}{$\mathrm{BE}(1,0.5)$} & ${ }_{\omega} \sigma_{i}=0.05$ & $3.7755^{*} 10^{-6}$ & $4.8813^{*} 10^{-3}$ & $3.1662^{*} 10^{-6}$ & $5.4007^{*} 10^{-3}$ & $1.4122^{*} 10^{-2}$ \\
\hline & ${ }_{\omega} \sigma_{i}=0.10$ & $1.4818^{*} 10^{-5}$ & $1.9343^{*} 10^{-2}$ & $1.1618^{*} 10^{-5}$ & $2.0172^{*} 10^{-2}$ & $5.5211^{*} 10^{-2}$ \\
\hline & ${ }_{\omega} \sigma_{i}=0.15$ & $3.3855^{*} 10^{-5}$ & $6.4107^{*} 10^{-2}$ & $2.9979 * 10^{-5}$ & $6.4740^{*} 10^{-2}$ & $1.8183^{*} 10^{-1}$ \\
\hline \multirow[t]{3}{*}{$\mathrm{TW}(20)$} & $\omega \sigma_{i}=0.05$ & $1.1022^{*} 10^{-4}$ & 2.5203 & $1.9994^{*} 10^{-6}$ & 3.5076 & 6.1608 \\
\hline & ${ }_{\omega} \sigma_{i}=0.10$ & $1.1130^{*} 10^{-4}$ & 2.5217 & $2.6444^{*} 10^{-6}$ & 3.5249 & 6.3360 \\
\hline & ${ }_{\omega} \sigma_{i}=0.15$ & $1.1872^{*} 10^{-4}$ & 2.5613 & $3.0510^{*} 10^{-6}$ & 3.5735 & 6.4498 \\
\hline
\end{tabular}



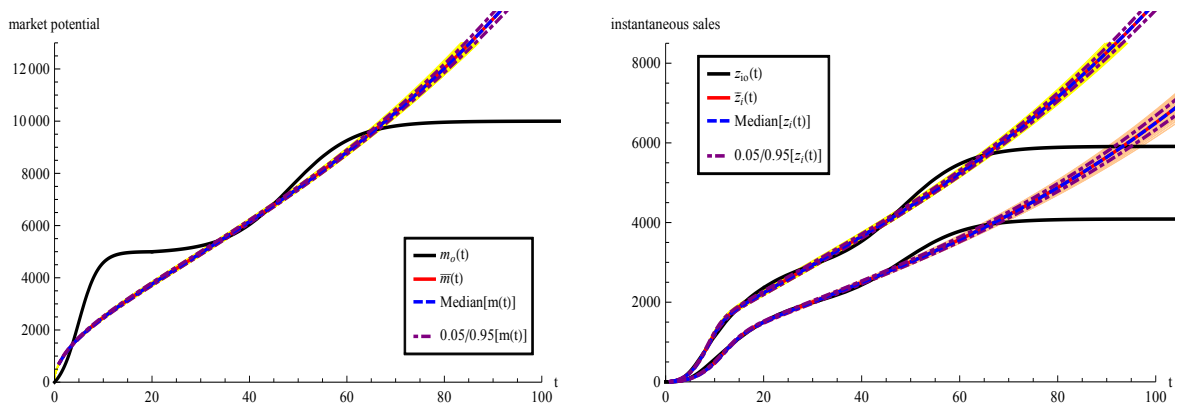

FIG A5.20. Case TW(20), ${ }_{\omega} \sigma_{i}=0.05$. Left side: $\hat{m}_{k}(t)$ (yellow trajectories), true $m_{0}(t)$, $\bar{m}(t)=\frac{1}{1000} \sum_{k=1}^{1000} \hat{m}_{k}(t)$, median trajectory, and quantile trajectories (0.05 and 0.95). Right side: $\hat{z}_{i k}(t)$ (yellow and orange trajectories), true $z_{i 0}(t), \bar{z}_{i}(t)=\frac{1}{1000} \sum_{k=1}^{1000} \hat{z}_{i k}(t)$, median trajectory, and quantile trajectories (0.05 and 0.95).
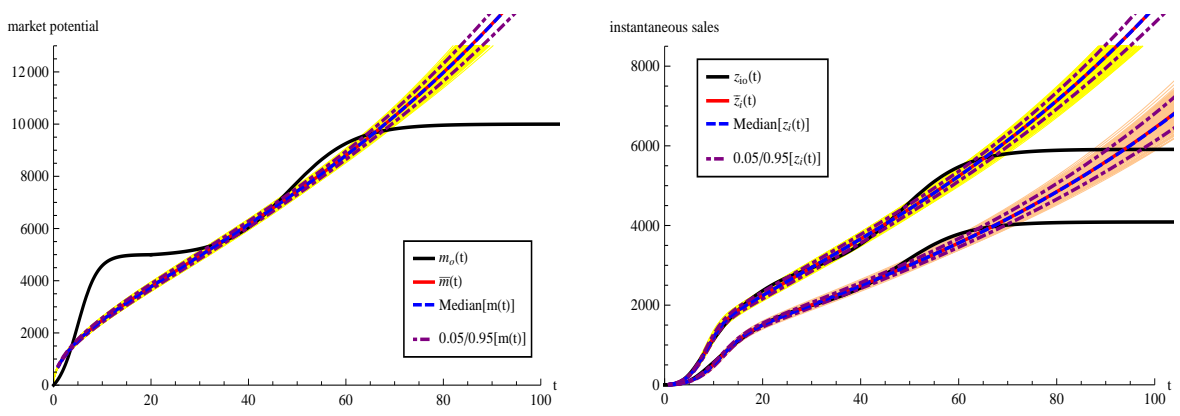

FIG A5.21. Case TW(20), ${ }_{\omega} \sigma_{i}=0.10$. Left side: $\hat{m}_{k}(t)$ (yellow trajectories), true $m_{0}(t)$, $\bar{m}(t)=\frac{1}{1000} \sum_{k=1}^{1000} \hat{m}_{k}(t)$, median trajectory, and quantile trajectories (0.05 and 0.95). Right side: $\hat{z}_{i k}(t)$ (yellow and orange trajectories), true $z_{i 0}(t), \bar{z}_{i}(t)=\frac{1}{1000} \sum_{k=1}^{1000} \hat{z}_{i k}(t)$, median trajectory, and quantile trajectories (0.05 and 0.95).

alternative $m(t)$ specifications. We can see that when $m(t)$ belongs to the Bemmaor family, the misspecification has a very small impact on the precision of the evolutionary parameters. For two-wave $m(t)$, the impact is larger.

\section{References.}

Bemmaor, A. C. (1994). Modeling the diffusion of new durable goods: Word-of-mouth effect versus consumer heterogeneity. In Research Traditions in Marketing (G. Laurent, G. L. Lilien and B. Pras, eds.) 201-229. Kluwer Academic, Boston, MA.

Bemmaor, A. C. and LeE, J. (2002). The impact of heterogeneity and ill-conditioning on diffusion model parameter estimates. Marketing Science 21 209-220.

Boswijk, H. P. and Franses, P. H. (2005). On the Econometrics of the Bass Diffusion Model. Journal of Business and Economic Statistics 23 255-268.

Guseo, R. and Guidolin, M. (2009). Modelling a dynamic market potential: a class of Automata Networks for diffusion of innovations. Technological Forecasting and Social 

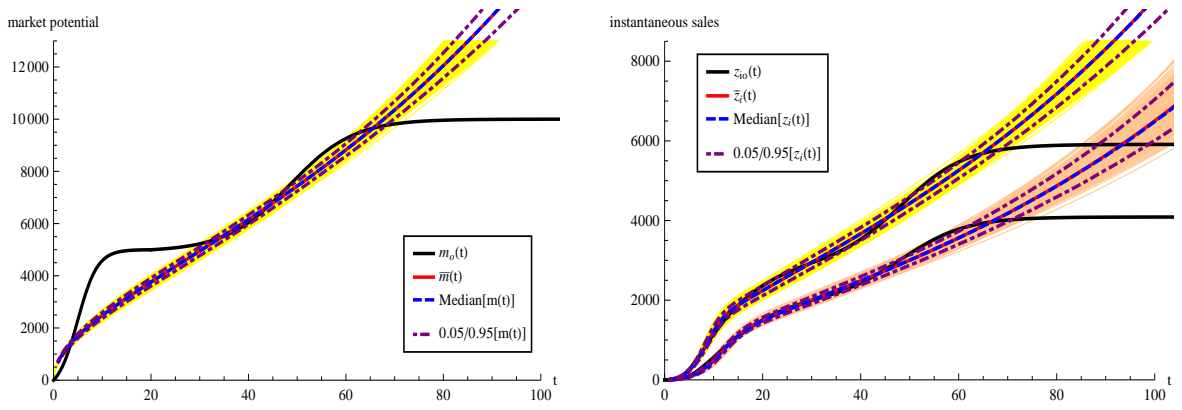

FIG A5.22. Case $T W(20),{ }_{\omega} \sigma_{i}=0.15$. Left side: $\hat{m}_{k}(t)$ (yellow trajectories), true $m_{0}(t)$, $\bar{m}(t)=\frac{1}{1000} \sum_{k=1}^{1000} \hat{m}_{k}(t)$, median trajectory, and quantile trajectories (0.05 and 0.95). Right side: $\hat{z}_{i k}(t)$ (yellow and orange trajectories), true $z_{i 0}(t), \bar{z}_{i}(t)=\frac{1}{1000} \sum_{k=1}^{1000} \hat{z}_{i k}(t)$, median trajectory, and quantile trajectories (0.05 and 0.95).

Change 76 806-820.

Guseo, R. and Mortarino, C. (2014). Within-brand and cross-brand word-of-mouth for sequential multi-innovation diffusions. IMA Journal of Management Mathematics 25 $287-311$.

Karmeshu, and Goswami, D. (2001). Stochastic evolution of innovation diffusion in heterogeneous groups: Study of life cycle patterns. IMA Journal of Management Mathematics 12 107-126.

Seber, G. A. F. and Wild, C. J. (2003). Nonlinear Regression. Wiley, New York.

Srinivasan, V. and Mason, C. H. (1986). Nonlinear least squares estimation of new product diffusion models. Marketing Science 5 169-178.

Department of Statistical Sciences
University of Padova, Italy

E-MAIL: renato.guseo@unipd.it; mortarino@stat.unipd.it 